\title{
Higgs + Multi-jets in Gluon Fusion
}

\author{
Nicolas Greiner* \\ Physik-Institut, Universität Zürich, Wintherturerstrasse 190, CH-8057 Zürich, Switzerland \\ E-mail: greinerephysik.uzh.ch
}

\section{Stefan Höche}

SLAC National Accelerator Laboratory, Menlo Park, CA 94025, USA

E-mail: shoecheeslac.stanford.edu

\section{Gionata Luisoni}

Theoretical Physics Department, CERN, Geneva, Switzerland

E-mail: gionata.luisoni@cern.ch

\section{Marek Schönherr}

Physik-Institut, Universität Zürich, Wintherturerstrasse 190, CH-8057 Zürich, Switzerland

E-mail: marek.schoenherraphysik.uzh.ch

\section{Jan-Christopher Winter}

Department of Physics and Astronomy, Michigan State University, East Lansing, MI 48824,

U.S.A.

E-mail: jwinter@cern.ch

\section{Valery Yundin}

Max-Planck-Institut für Physik, Föhringer Ring 6, D-80805 München, Germany

E-mail: yundin@mpp.mpg.de

We present a detailed phenomenological analysis of the production of a Standard Model Higgs boson in association with up to three jets. The Higgs is produced via gluon fusion, which is an irreducible background to the vector boson fusion mechanism. We calculate the next-to-leading order corrections in QCD in the limit of an infinitely heavy top quark. Numerical results are presented for a large variety of observables, for different selection cuts, and for different choices of the tagging scheme.

SLAC-PUB-16455, ZU-TH-1/16

12th International Symposium on Radiative Corrections (Radcor 2015) and LoopFest XIV (Radiative Corrections for the LHC and Future Colliders)

15-19 June, 2015

UCLA Department of Physics \& Astronomy Los Angeles, USA

\footnotetext{
*Speaker.
} 


\section{Introduction}

A major step after the discovery of the Higgs particle $[1,2]$ is the precise determination of its nature. This includes its couplings to fermions and bosons. As different models lead to various deviations from the Standard Model prediction a precise prediction for the Standard Model contribution is an essential step to the revelation of the underlying mechanism of electroweak symmetry breaking. During Run II at the LHC, the vector boson fusion mechanism will play a leading role. In this production mode, a Higgs boson is created by annihilation of virtual $W$ or $Z$ bosons, radiated off the initial-state (anti-)quarks in a $t$-channel scattering process with no color exchange at leading order $[3,4]$. It allows for a direct acces to the couplings between the Higgs and the electroweak gauge bosons while at the same time providing a clean signature with two forward jets with only little hadronic energy between these tagging jets.

The main production mechanism is however given by the gluon fusion channel where the Higgs is produced out of two initial state gluons via a loop of heavy quarks. The gluon fusion mechanism constitutes an interesting process on its own, but it is also an irreducible background to the VBF channel. In both cases a reliable theoretical prediction is indispensable. In this talk we discuss the calculation and the phenomenlogy of the production of a Standard Model Higgs boson in association up to three jets, as described in detail in Refs. [5-7]. The calculation takes into account next-to-leading order QCD corrections and it is carried out in the limit of an infinitely heavy top quark. We discuss two different set of cuts, namely a set of basic cuts that are suitable for the gluon fusion contribution and a more restrictive set of cuts which is more suited for VBF analyses. In addition we discuss different schemes for defining the tagging jets.

\section{Calculational setup}

We perform the calculation of the NLO corrections by using the automated tools GoSAM $[8,9]$ for the generation of the virtual amplitudes, and SHERPA [10] for the tree-level like amplitudes, subtraction terms and phase space integration. The two are linked using the Binoth Les Houches Accord [11,12], a standard interface for event and parameter passing between one-loop programs and Monte Carlo generators.

The one-loop program GoSAM is based on an algebraic generation of $d$-dimensional integrands using a Feynman diagrammatic approach, employing QGRAF [13] and FORM [14,15] for the diagram generation, and SPINNEY [16], HAGgIES [17] and FORM to write an optimized Fortran output. For the reduction of the tensor integrals, we used NINJA [26-28], which is an automated package carrying out the integrand reduction via Laurent expansion. Alternatively, one can use other reduction techniques such as the standard OPP method [18-20] as implemented in SAMURAI [21] or methods of tensor integral reduction as implemented in GOLEM95 [22-24]. The resulting scalar integrals are evaluated using ONELOOP [25]. More details on the calculation and reduction of multi-loop integrands have been presented at this workshop by Giovanni Ossola in Ref. [41].

The calculation of tree-level matrix elements real emission contribution as well as the subtraction terms in the Catani-Seymour approach [36] has been done within SHERPA [10] using the matrix element generator COMIX $[29,30]$. We have validated the result obtained by COMIX with the results we have obtained in Ref [6], where we have used a combination of MADGRAPH 4 [31,32], 
MAdDipole [33,34] and MAdEvent [35] for the calculation of real emission matrix elements, subtraction terms and the phase space integration of the real emission contribution.

\subsection{Cuts and parameter settings}

We have produced results for two center of mass energies at 8 and $13 \mathrm{TeV}$. In both cases we have applied two sets of cuts, one baseline set which is based on a minimal set of cuts to render the cross section finite, and a more resitrictive set of cuts which is typically used in the context of VBF searches. In both cases jets are clustered using the anti- $k_{T}$ algorithm $[37,38]$ as implemented in the FASTJET package [39]. If not specified explicitly, the jet radius and PDF set have been set to $R=0.4$ and CT10nlo [40], respectively. The baseline set consists of the following cuts:

$$
p_{T}>30 \mathrm{GeV}, \quad|\eta|<4.4 .
$$

In the VBF case two additional cuts have been imposed, given by

$$
m_{j_{1} j_{2}}>400 \mathrm{GeV}, \quad\left|\Delta y_{j_{1}, j_{2}}\right|>2.8 .
$$

Here the two jets $j_{1}$ and $j_{2}$ denote the tagging jets. Their selection is not unique and we study two different schemes, one where the two tagging jets are the two jets with the highest $p_{T}$ ( $p_{T}$-tagging), and one where the most forward and most backward jet (in rapidity) yield the two tagging jets (y-tagging).

The central scale for the variation of remormalization and factorization scale is chosen to be

$$
\mu_{F}=\mu_{R} \equiv \frac{\hat{H}_{T}^{\prime}}{2}=\frac{1}{2}\left(\sqrt{m_{H}^{2}+p_{T, H}^{2}}+\sum_{i}\left|p_{T, i}\right|\right) \text {. }
$$

However it is not obvious whether this dynamical scale is also a good choice to be used for the gluon-gluon-Higgs coupling. One might argue that the Higgs mass is the appropriate scale there. Therfore we consider three different scale choices, defined as

$$
\begin{array}{ll}
\text { A : } & \alpha_{\mathrm{s}}\left(x \cdot \frac{\hat{H}_{T}^{\prime}}{2}\right)^{3} \alpha_{\mathrm{s}}\left(x \cdot m_{H}\right)^{2}, \\
\mathrm{~B}: & \alpha_{\mathrm{s}}\left(x \cdot \frac{\hat{H}_{T}^{\prime}}{2}\right)^{5}, \\
\mathrm{C}: & \alpha_{\mathrm{s}}\left(x \cdot m_{H}\right)^{5} .
\end{array}
$$

The presence of the factor $x$ indicates that this scale is varied in the range $x \in[0.5 \ldots 2]$.

\section{Numerical results for gluon fusion setup}

We start the discussion of the numerical results with the basic gluon fusion cuts. One of the most important obserables is the total cross section. In Figure 1 we show the total cross sections for both leading order and next-to-leading order for the processes $\mathrm{H}+1$ jet, $\mathrm{H}+2$ jets and $\mathrm{H}+3$ jets at $E_{\mathrm{cm}}=8 \mathrm{TeV}$ (left plot) and $E_{\mathrm{cm}}=13 \mathrm{TeV}$ (right plot). The result have been obtained for the three 

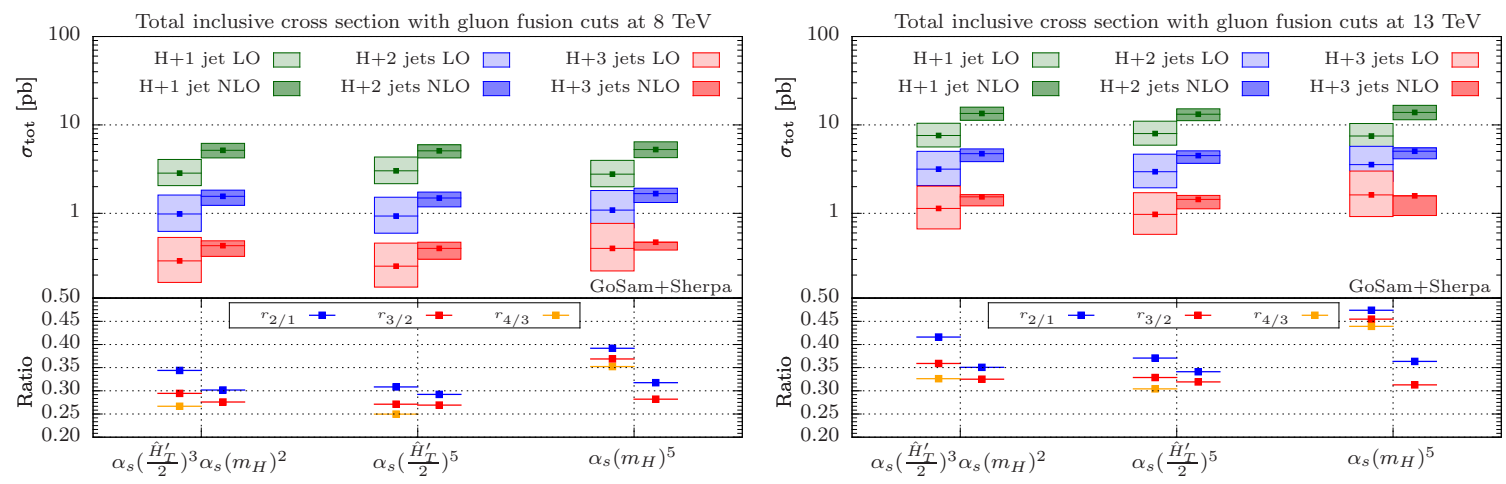

Figure 1: Total cross sections at LO (left side of each column) and NLO (right side of each column) for $\mathrm{H}+1$ jet (green), $\mathrm{H}+2$ jets (blue) and $\mathrm{H}+3$ jets (red) production using the three different scale choices as explained in text. In the lower part of the plots, the ratios $r_{2 / 1}$ (blue), $r_{3 / 2}$ (red) and $r_{4 / 3}$ (orange) are shown. Results have been obtained for $8 \mathrm{TeV}$ and $13 \mathrm{TeV}$ (left and right plot respectively).

scale choices described above. On the level of total cross section one only observes a mild dependence on the scale choice, in particular for the NLO results. For the fixed scale one observers an enhancement of the LO ratios. An increase of the center of mass energy from 8 to $13 \mathrm{TeV}$ also only as a small influence on the overall pattern. The situation is different when one looks at differential distributions. In Figure 2 we show the transverse momentum distribution for the Higgs boson for the $\mathrm{H}+3$ jets process at a center of mass energy of $8 \mathrm{TeV}$. The subplots show the distribution for the three different scale choices A, B and C, while Fig. 2a shows the results for the different scales normalized to the NLO result for scale A. The advantage of scale B is the flatness of the $K$-factor over the entire $p_{T}$ range. This supports our choice to make scale B the default scale. For the lower $p_{T}$ region up to $\sim 250 \mathrm{GeV}$, scale $\mathrm{C}$ seems to be a sensible choice as well. However, it completely breaks down for higher $p_{T}$, and the $K$-factor can even become negative. Further support for using scale $\mathrm{B}$ as the default choice comes from looking at the $p_{T}$ distribution of the 'wimpiest' jet for each multiplicity. This means looking at the first jet for $\mathrm{H}+1$ jet, at the second jet for $\mathrm{H}+2$ jets, and at the third jet for $\mathrm{H}+3$ jets. This is illustrated in Figure 3. The left hand side shows the $p_{T}$ of the three jets for scale choice B, the right hand side shows scale choice A. The lower part of the plots show the ratio of NLO versus LO for each jet. For better visibility the ratios are multiplied with a certain factor to avoid overlap of the bands of the different jets. As can be seen from the ratio plots, the purely dynamical scale choice B leads to flat K-factors, whereas scale choice A shows a decrease of the k-factor with increasing transverse momentum.

Another interesting question is how observables that are defined independent of a certain jet multiplicity, like the transverse momentum of the Higgs, changes under the presence of additional QCD radiation. The upper plots show the NLO distributions for one, two and three jets (which we have obtained from the one-jet, two-jet and three-jet NLO calculations, respectively). Unless stated otherwise, the jet multiplicity is exclusive, labelled by 'excl', i.e. a veto on any additional jet activity is in place. The 1-jet and the 3-jet processes are shown twice, once for the exclusive case, and once for the inclusive case, labelled by 'incl'. The lower subpanels show each contribution normalized to the inclusive prediction of the core process, i.e. the most inclusive one, here given by the $\mathrm{H}+1$ 


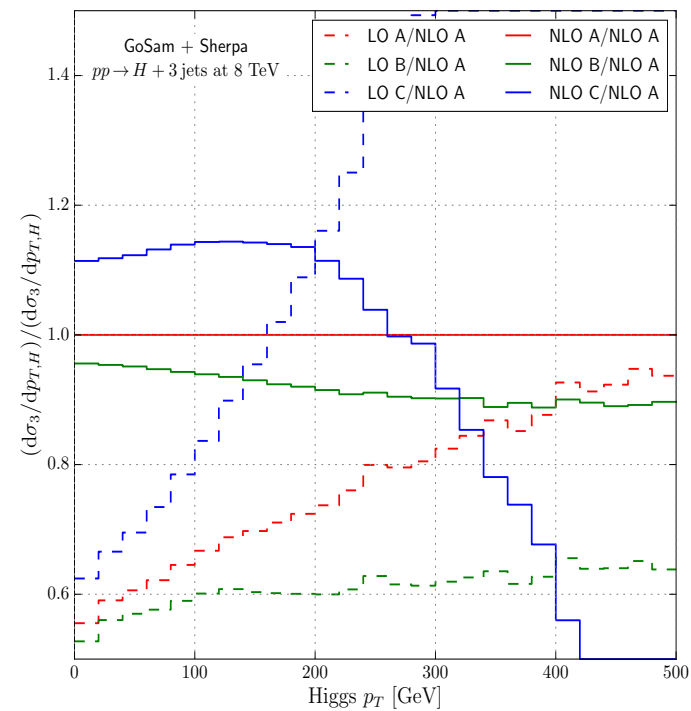

(a) Ratio

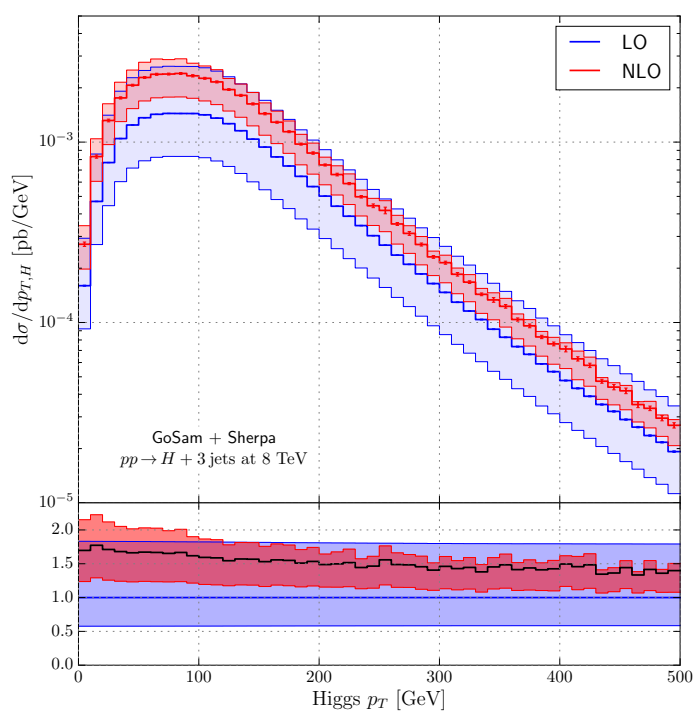

(c) Scale choice B

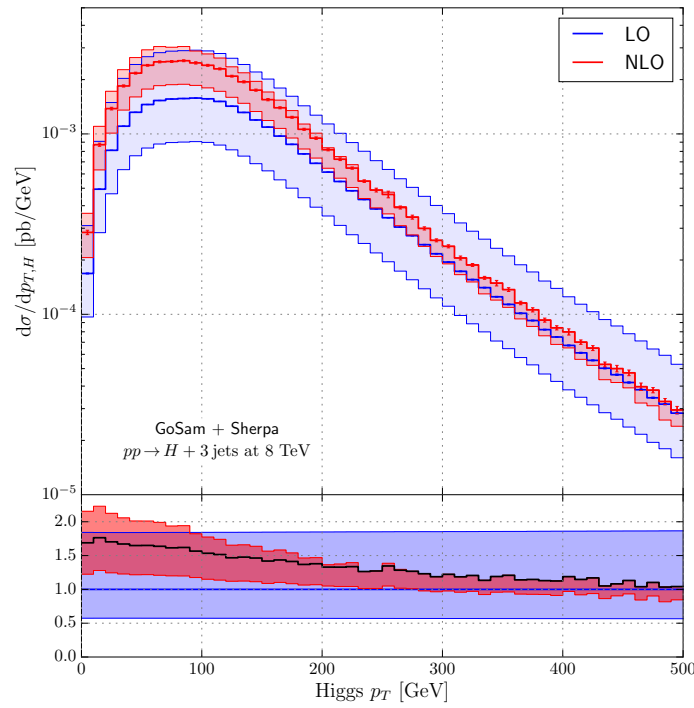

(b) Scale choice A

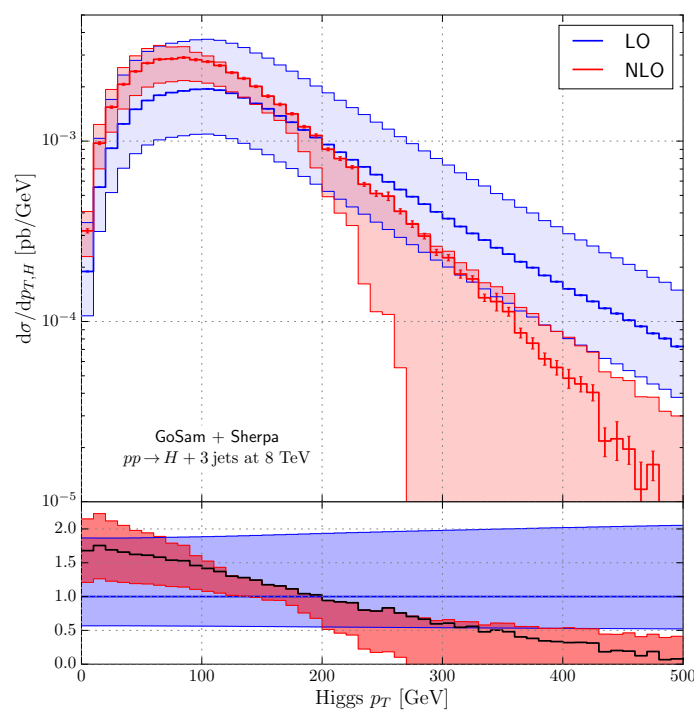

(d) Scale choice C

Figure 2: The $p_{T}$ distribution of the Higgs boson in $\mathrm{H}+3$ jets production at the $8 \mathrm{TeV}$ LHC presented for the three scales A, B and C. The subplot 2a shows the same central predictions normalized to the NLO result for scale A. Each ratio plot depicts the respective differential $K$-factors and their envelopes obtained from scale variations at LO and NLO.

jet process. The plots in the middle and lower panel are constructed following the same principle but using the NLO core process of increased jet multiplicity, namely $\mathrm{H}+2$ jets and $\mathrm{H}+3$ jets, respectively. The middle row of Fig. 4 hence depicts the same situation but without accounting for the $\mathrm{H}+1$ jet process; and, for the lower row, there are only two distributions left to show, the ones for the exclusive as well as the inclusive $\mathrm{H}+3$ jets process. One can see that the low energy region 

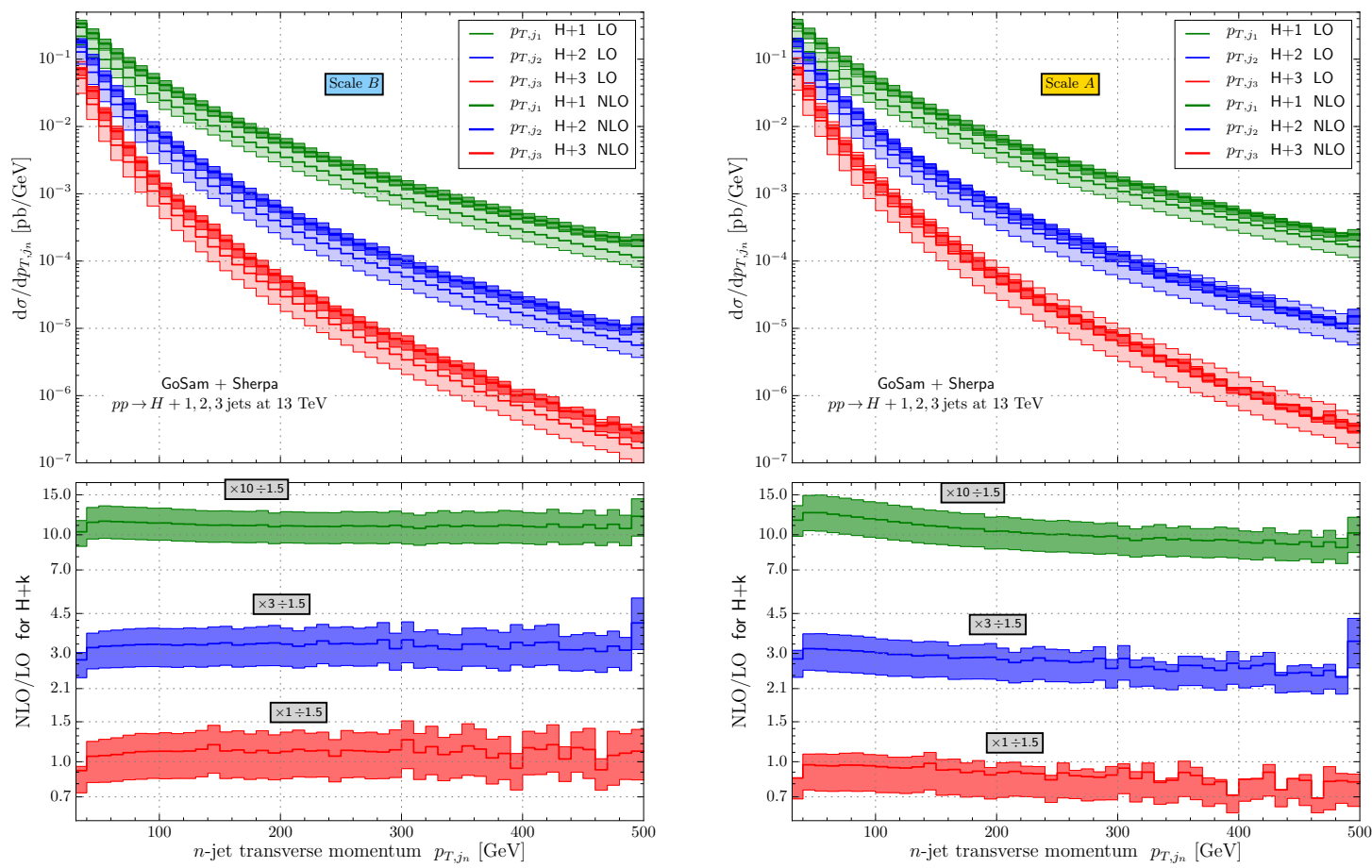

Figure 3: Transverse momentum distribution of the 'wimpiest' jet in $\mathrm{H}+n$ jets production at the LHC. Using $p_{T}$ ordering the first, second and third leading jet are shown in $\mathrm{H}+1$ jet, $\mathrm{H}+2$ jets and $\mathrm{H}+3$ jets at $13 \mathrm{TeV}$, respectively; on the left with the default scale choice $\mathrm{B}$, on the right with the scale choice $\mathrm{A}$.
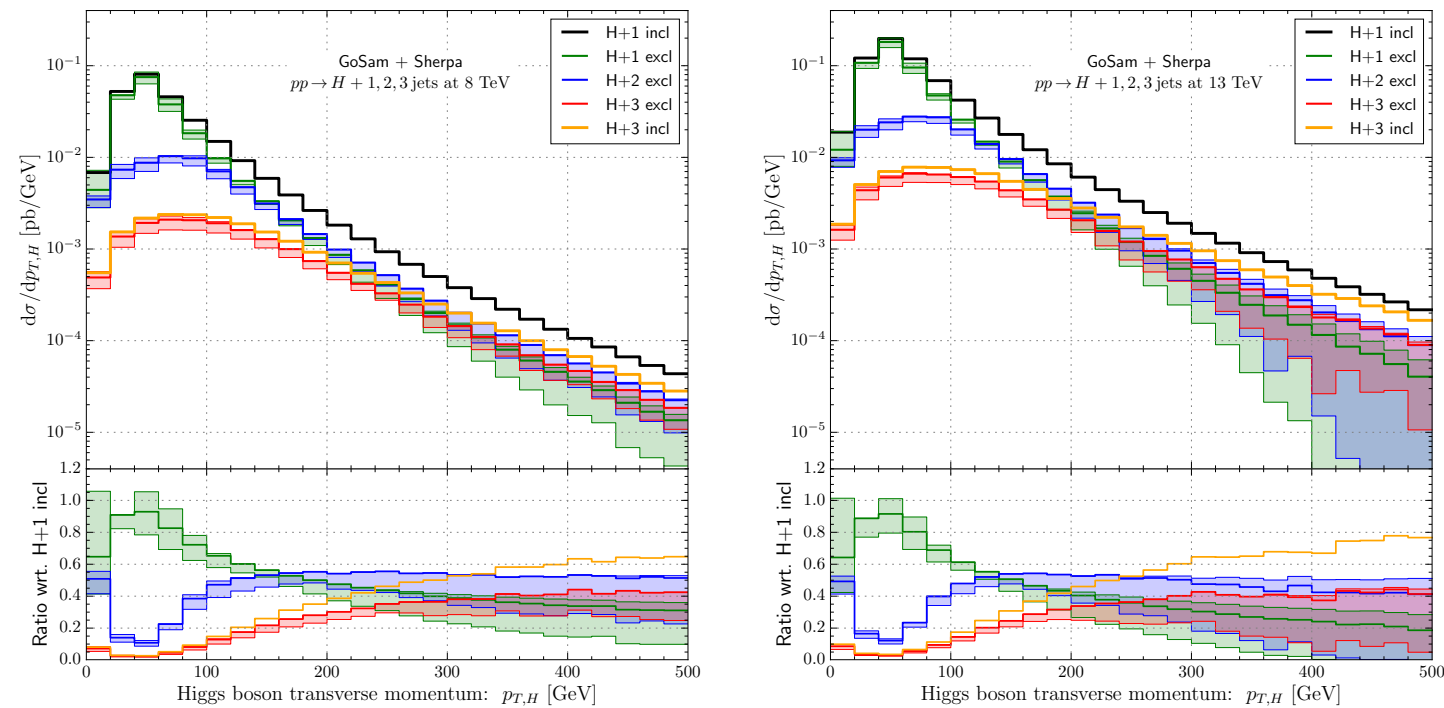

Figure 4: Transverse momentum distribution of the Higgs for the different jet multiplicities. L.h.s shows the result for $8 \mathrm{TeV}$, r.h.s shows the distribution for $13 \mathrm{TeV}$.

is dominated by the exclusive $\mathrm{H}+1$ jet contribution. The $\mathrm{H}+2$ jets contribution is negligible in that region, however starts to dominate already in a region above approx. $200 \mathrm{GeV}$. Going further up 

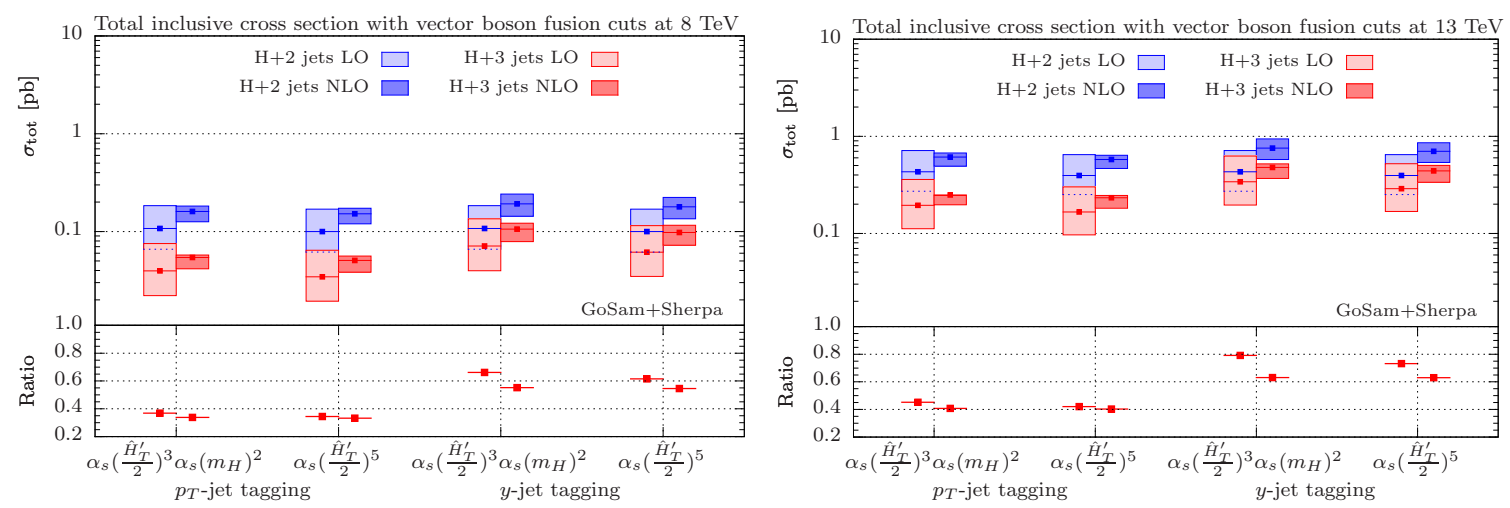

Figure 5: Total cross sections at $\mathrm{LO}$ and $\mathrm{NLO}$ for $\mathrm{H}+2$ jets (blue) and $\mathrm{H}+3$ jets (red) using VBF kinematical cuts and two different tagging jet definitions. Results are shown for the two scale choices $\mathrm{A}$ and $\mathrm{B}$, as well as the two energies of $8 \mathrm{TeV}$ (left plot) and $13 \mathrm{TeV}$ (right plot). The lower part of each plot depicts the inclusive cross section ratios $r_{3 / 2}$ for the different scales and tag jet approaches.

in the spectrum increases the $\mathrm{H}+3$ jets contribution which will eventuall dominate the spectrum. In other words, at high enough energies, to have one jet more comes with the same or even higher probability. This has to be kept in mind when comparing an inclusive measurement with a fixed order calculation of a given number of jets. For low multiplicities the description becomes inaccurate already at relatively low energies of around $200 \mathrm{GeV}$. A theoretical prediction that is based on a merged result of different multiplicities will yield a better description of the data.

\section{Phenomenology with vector boson fusion cuts}

Gluon fusion is an irreducible background to the VBF channel, the challenging task for theory is therefore to provide a precise prediction of its rate compared to the signal. In this section we discuss the results obtained from the gluon fusion contribution under the presence of the additional VBF cuts described in section 2.1. Again we start the discussion with the total cross section, for the VBF selection we now also consider the differences betwee the two tagging schemes described above, $p_{T}$-tagging and $y$-tagging. The total cross section for the different energies, scales and tagging scheme is shown in Figure 5. Having ruled out the fixed scale as a sensible choice we only show the result for the two scales A and B. Also in the case of VBF selection the differences between the scale choices are rather small. The tagging scheme has a much bigger impact. The $y$-tagging increases the ratios which means that it increases the fraction of the processes with higher multiplicity. The impact of the higher jet multiplicities can be understood by looking at the exclusive $n$-jet cross section. This is shown for $\mathrm{H}+2$ jets and $\mathrm{H}+3$ jets in Figure 6 . In the upper row the results are shown using $p_{T}$ tagging, the lower row displays the $n$ jet cross section after applying the $y$-tagging scheme. In both cases, but particularly for the $y$-tagging one sees that the real emission contribution constitutes a substantial fraction of the total cross section. This also means that a large fraction of the cross section is only described at leading order accuracy. This stresses the importance of the inclusion of NLO results with higher multiplicities into the theoretical prediction. 

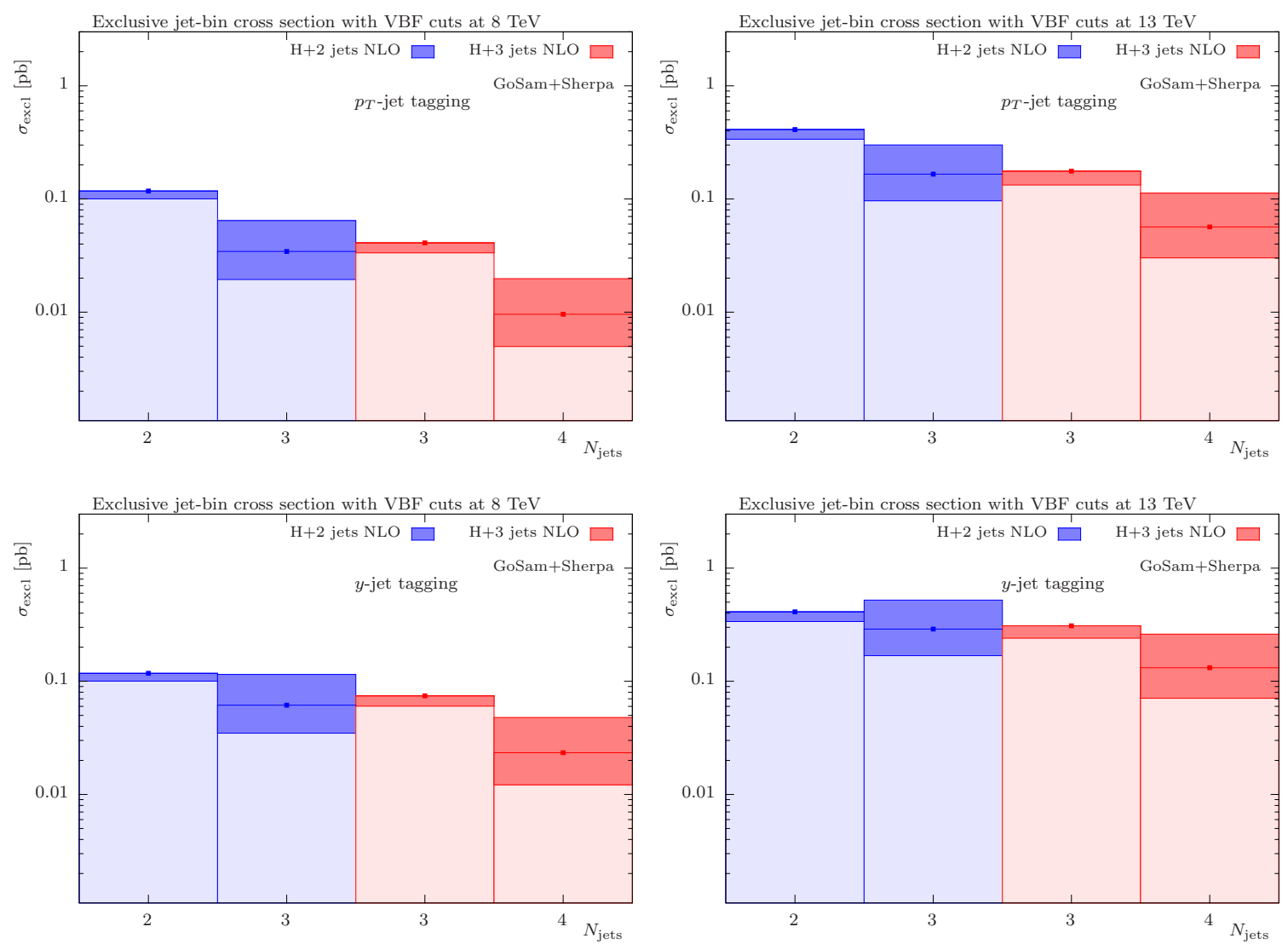

Figure 6: Exclusive jet cross sections in $\mathrm{H}+2$ jets and $\mathrm{H}+3$ jets production at the $8 \mathrm{TeV}$ (left) and $13 \mathrm{TeV}$ (right) LHC after application of typical VBF selection constraints (using scale choice B). The upper and lower set of plots display the results based on different jet-tagging strategies, namely for $p_{T}$ jet-tagging and $y$ jet-tagging, respectively.

\section{Conclusions}

In this talk we presented phenomenological results for the production of a Standard Model Higgs boson via the gluon fusion mechanism in the heavy top mass limit in association with up to three jets. We investigated the role of the scale choice as well as the effects for different set of cuts, also allowing to assess the role of the gluon fusion contribution in VBF searches. Furthermore we discussed a variety of important observables allowing for a better discrimination between the gluon fusion and vector boson fusion contribution. Further improvements could certainly be achieved by providing a merged NLO result of the different jet multiplicities, but also through the inclusion of top-quark mass effects as well as the matching of the $\mathrm{H}+3$ jets NLO result with a parton shower. Due to the complexity of these improvements they are however beyond the scope of this paper and we leave them for future work.

\section{Acknowledgments}

We would like to thank the members of the GoSam collaboration for their help and effort. This work was supported by the Swiss National Science Foundation under contracts PZ00P2_154829 
and PP00P2-128552 and by the US Department of Energy under contract DE-AC02-76SF00515.

\section{References}

[1] ATLAS Collaboration Collaboration, G. Aad et al., Observation of a new particle in the search for the Standard Model Higgs boson with the ATLAS detector at the LHC, Phys.Lett. B716 (2012) 1-29, [arXiv:1207.7214].

[2] CMS Collaboration Collaboration, S. Chatrchyan et al., Observation of a new boson at a mass of 125 GeV with the CMS experiment at the LHC, Phys.Lett. B716 (2012) 30-61, [arXiv: 1207.7235 ].

[3] R. Cahn and S. Dawson, Production of Very Massive Higgs Bosons, Phys.Lett. B136 (1984) 196.

[4] G. L. Kane, W. Repko, and W. Rolnick, The Effective W+-, Z0 Approximation for High-Energy Collisions, Phys.Lett. B148 (1984) 367-372.

[5] N. Greiner, S. Hoeche, G. Luisoni, M. Schonherr, J. C. Winter and V. Yundin, arXiv:1506.01016 [hep-ph].

[6] G. Cullen et al., Phys. Rev. Lett. 111, no. 13, 131801 (2013) doi:10.1103/PhysRevLett.111.131801 [arXiv:1307.4737 [hep-ph]].

[7] H. van Deurzen et al., Phys. Lett. B 721, 74 (2013) doi:10.1016/j.physletb.2013.02.051 [arXiv:1301.0493 [hep-ph]].

[8] G. Cullen, N. Greiner, G. Heinrich, G. Luisoni, P. Mastrolia, et al., Automated One-Loop Calculations with GoSam, Eur.Phys.J. C72 (2012) 1889, [arXiv:1111.2034].

[9] G. Cullen, H. van Deurzen, N. Greiner, G. Heinrich, G. Luisoni, et al., GOSAM-2.0: a tool for automated one-loop calculations within the Standard Model and beyond, Eur.Phys.J. C74 (2014), no. 8 3001, [arXiv:1404.7096].

[10] T. Gleisberg, S. Höche, F. Krauss, M. Schönherr, S. Schumann, et al., Event generation with SHERPA 1.1, JHEP 0902 (2009) 007, [arXiv: 0811 . 4622].

[11] T. Binoth, F. Boudjema, G. Dissertori, A. Lazopoulos, A. Denner, et al., A Proposal for a standard interface between Monte Carlo tools and one-loop programs, Comput.Phys.Commun. 181 (2010) 1612-1622, [arXiv:1001.1307].

[12] S. Alioli, S. Badger, J. Bellm, B. Biedermann, F. Boudjema, et al., Update of the Binoth Les Houches Accord for a standard interface between Monte Carlo tools and one-loop programs, Comput.Phys.Commun. 185 (2014) 560-571, [arXiv:1308.3462].

[13] P. Nogueira, Automatic Feynman graph generation, J.Comput.Phys. 105 (1993) 279-289.

[14] J. Vermaseren, New features of FORM, math-ph/0010025.

[15] J. Kuipers, T. Ueda, J. Vermaseren, and J. Vollinga, FORM version 4.0, Comput.Phys.Commun. 184 (2013) 1453-1467, [arXiv:1203.6543].

[16] G. Cullen, M. Koch-Janusz, and T. Reiter, Spinney: A Form Library for Helicity Spinors, Comput.Phys.Commun. 182 (2011) 2368-2387, [arXiv: 1008 . 0803 ].

[17] T. Reiter, Optimising Code Generation with haggies, Comput.Phys.Commun. 181 (2010) 1301-1331, [arXiv:0907.3714].

[18] G. Ossola, C. G. Papadopoulos, and R. Pittau, Reducing full one-loop amplitudes to scalar integrals at the integrand level, Nucl.Phys. B763 (2007) 147-169, [hep-ph/ 0609007 ]. 
[19] P. Mastrolia, G. Ossola, C. Papadopoulos, and R. Pittau, Optimizing the Reduction of One-Loop Amplitudes, JHEP 0806 (2008) 030, [arXiv: 0803.3964 ].

[20] G. Ossola, C. G. Papadopoulos, and R. Pittau, On the Rational Terms of the one-loop amplitudes, JHEP 0805 (2008) 004, [arXiv: 0802 . 1876 ].

[21] P. Mastrolia, G. Ossola, T. Reiter, and F. Tramontano, Scattering AMplitudes from Unitarity-based Reduction Algorithm at the Integrand-level, JHEP 1008 (2010) 080, [arXiv: 1006.0710 ].

[22] G. Heinrich, G. Ossola, T. Reiter, and F. Tramontano, Tensorial Reconstruction at the Integrand Level, JHEP 1010 (2010) 105, [arXiv: 1008.2441$].$

[23] T. Binoth, J.-P. Guillet, G. Heinrich, E. Pilon, and T. Reiter, Golem95: A Numerical program to calculate one-loop tensor integrals with up to six external legs, Comput.Phys.Commun. 180 (2009) 2317-2330, [arXiv:0810.0992].

[24] G. Cullen, J. P. Guillet, G. Heinrich, T. Kleinschmidt, E. Pilon, et al., Golem95C: A library for one-loop integrals with complex masses, Comput.Phys.Commun. 182 (2011) 2276-2284, [arXiv:1101.5595].

[25] A. van Hameren, OneLOop: For the evaluation of one-loop scalar functions, Comput.Phys.Commun. 182 (2011) 2427-2438, [arXiv: 1007 .4716].

[26] P. Mastrolia, E. Mirabella, and T. Peraro, Integrand reduction of one-loop scattering amplitudes through Laurent series expansion, JHEP 1206 (2012) 095, [arXiv:1203.0291].

[27] H. van Deurzen, G. Luisoni, P. Mastrolia, E. Mirabella, G. Ossola, et al., Multi-leg One-loop Massive Amplitudes from Integrand Reduction via Laurent Expansion, JHEP 1403 (2014) 115, [arXiv:1312.6678].

[28] T. Peraro, Ninja: Automated Integrand Reduction via Laurent Expansion for One-Loop Amplitudes, arXiv:1403.1229.

[29] T. Gleisberg and S. Höche, Comix, a new matrix element generator, JHEP 0812 (2008) 039, [arXiv:0808.3674].

[30] S. Höche, Efficient dipole subtraction with Comix, .

[31] T. Stelzer and W. Long, Automatic generation of tree level helicity amplitudes, Comput.Phys.Commun. 81 (1994) 357-371, [hep-ph/9401258].

[32] J. Alwall, P. Demin, S. de Visscher, R. Frederix, M. Herquet, et al., MadGraph/MadEvent v4: The New Web Generation, JHEP 0709 (2007) 028, [arXiv : 0706.2334 ].

[33] R. Frederix, T. Gehrmann, and N. Greiner, Automation of the Dipole Subtraction Method in MadGraph/MadEvent, JHEP 0809 (2008) 122, [arXiv: 0808 . 2128].

[34] R. Frederix, T. Gehrmann, and N. Greiner, Integrated dipoles with MadDipole in the MadGraph framework, JHEP 1006 (2010) 086, [arXiv: 1004 . 2905].

[35] F. Maltoni and T. Stelzer, MadEvent: Automatic event generation with MadGraph, JHEP 0302 (2003) 027, [hep-ph/0208156].

[36] S. Catani and M. Seymour, A General algorithm for calculating jet cross-sections in NLO QCD, Nucl.Phys. B485 (1997) 291-419, [hep-ph/9605323].

[37] M. Cacciari and G. P. Salam, Dispelling the $N^{3}$ myth for the $k_{t}$ jet-finder, Phys.Lett. B641 (2006) 57-61, [hep-ph/0512210]. 
[38] M. Cacciari, G. P. Salam, and G. Soyez, The Anti-k(t) jet clustering algorithm, JHEP 0804 (2008) 063, [arXiv:0802.1189].

[39] M. Cacciari, G. P. Salam, and G. Soyez, FastJet User Manual, Eur.Phys.J. C72 (2012) 1896, [arXiv:1111.6097].

[40] H.-L. Lai, M. Guzzi, J. Huston, Z. Li, P. M. Nadolsky, et al., New parton distributions for collider physics, Phys.Rev. D82 (2010) 074024, [arXiv: 1007.2241$].$

[41] Talk of Giovanni Ossola, in these proceedings 